\title{
Combined Algorithm of Image Registration Using Searching and Tracking Techniques
}

\author{
B. A. Alpatov, P. V. Babayan, M. V. Khosenko \\ Department of Automation and Information Technologies in Control, Ryazan State Radio \\ Engineering University, 59/1, Gagarin St., Ryazan, Russia, 390005
}

Keywords: image processing, transformation, registration.

\begin{abstract}
We analyze the problem of image registration and offer a new approach in which benefits of previously developed approaches are combined. The algorithm involves two techniques: searching and tracking. Feature detection and matching algorithms were applied to perform tracking technique. Correlation-extremal algorithm is used for searching technique. We describe the advantages of the presented idea in experimental results and give some examples which reflect the speed-up and the quality of registration.
\end{abstract}

\section{Introduction}

Registration process will be called matching of the corresponding pixels of two images. There are many problems and challenges in applied techniques of registering images including spatial reference images for remote sensing of the Earth, building panoramas of combined vision and others. Applying this technology in practice allows autonomous unmanned aerial vehicles, aircrafts and other aerial vehicles be coordinated and controlled. Hardware and software equipment also facilitates the direct control to the pilot, improves visual information on the devices. There are some methods of image registration, each possesses certain advantages and disadvantages and requires various pre-processed images. These methods can be divided into correlated and non-correlated. Images can be registered in various ways. One of the most preferred approaches is template matching. This approach includes calculation of the objective function, for example correlationextremal difference or some other kinds [9]. The disadvantage of it is high computation complexity. Other approaches use feature points and matching technologies [1-5]. But it's impossible to use them for registering images from different types of sources, for example TV and synthesized images. The goal of our work is to eliminate these problems by combining techniques together.

\section{System Overview}

\subsection{Discussed problem}

This article discusses the alternative decision of the problem of registration of VTM and TV images (TV image is an image from the video camera, VTM - virtual terrain model). The initial solution of this problem is based on the results obtained from matching their contour models using correlatedextremal algorithm (CEA). This algorithm is based on finding the extremum of the objective function between the contour models of the current image and VTM. This is described by the following rule:

$$
R(\Phi)=\sum_{x, y} M_{\Phi}(x, y) \cdot I(x, y),
$$

where $I(x, y)$ - contour model of the current TV image, $\Phi$ - vector of the observed position (shift, rotation, scale etc.). $M_{\Phi}(x, y)$ - image synthesized from VTM, using $\Phi$ vector. The metric is stored for each $\Phi$ in the result matrix $R(\Phi)$. $\Phi$ vector corresponding to the maximum of $R(\Phi)$ must be used to synthesize image that is registered with $I(x, y)$. 
The main disadvantage is high computational complexity especially when vector $\Phi$ contains more than three dimensions. To work correctly, comparable baseline data is required. We do not abandon this technique completely and just want to supplement and improve it. Therefore, it is proposed to use searching and tracking techniques to increase accuracy and processing performance of the video sequence. Algorithm with CEA is used in searching technique.

In tracking technique, registration process is based on using feature points of the image. Algorithms working with feature points are widely used in the problems of image processing and based on the allocation of a pair of feature points and further transformation of one image relative to the other. Full algorithm is shown on fig. 1. The developed algorithm includes searching and tracking techniques simultaneously. Firstly, searching technique is activated and transformed image of VTM is formed. It is subjected for further processing in a tracking technique. TV image involved in a searching technique is used as a base TV image. In a tracking technique solution of the problem of registering images is performed using geometric transformations to the previously obtained image of VTM. Estimation of the parameters of geometric transformations in this research is implemented by comparing the current TV image from the video sequence to the base TV image. If the similarity between base and acquired TV image decreases, it is necessary to perform searching registration again.

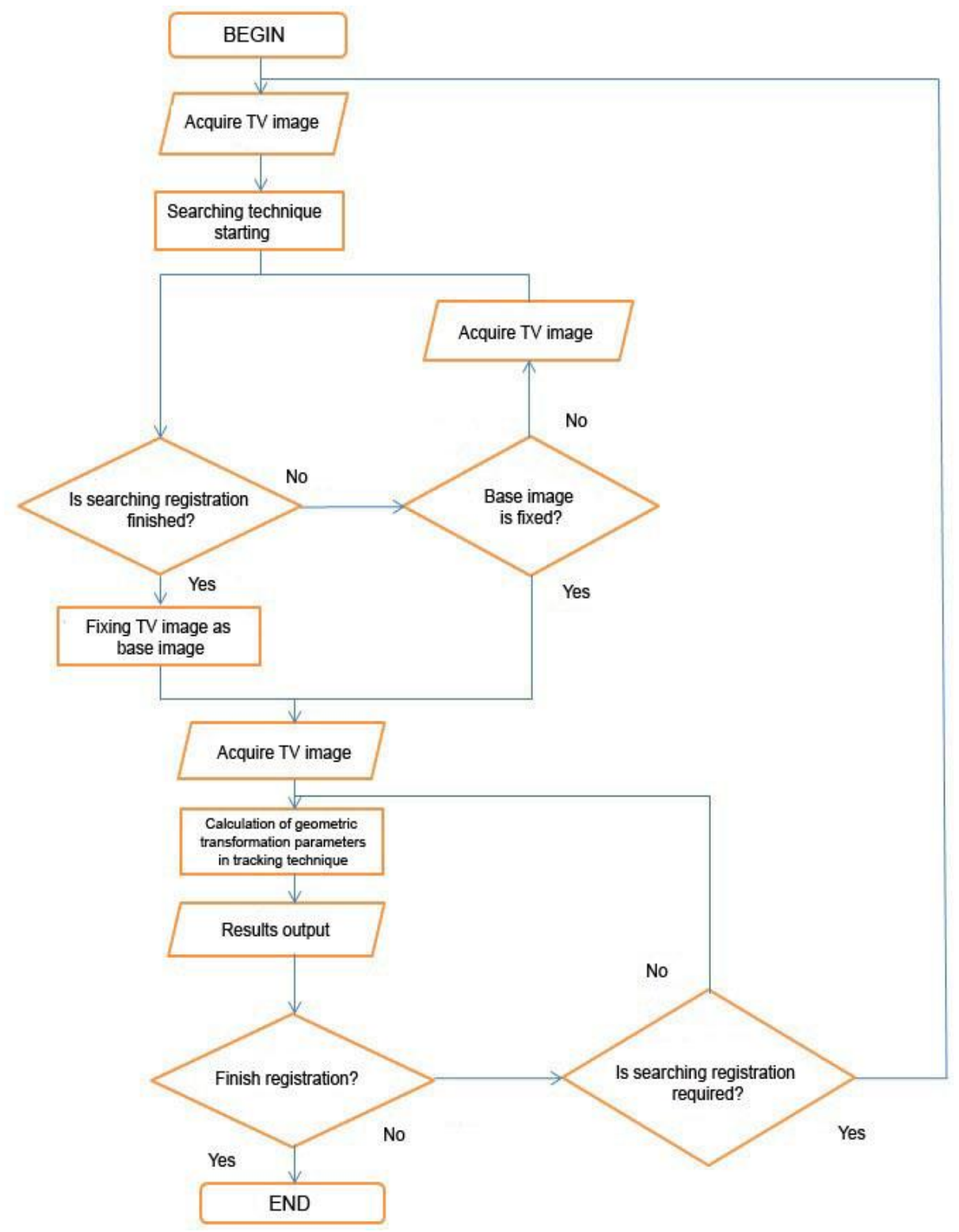

Figure 1. - Proposed algorithm. 


\subsection{Tracking technique}

The whole sequence of steps in a tracking technique is presented below:

- searching for the feature points, also called keypoints, then creation of their descriptors;

- matching feature points (creating matchers or inliers);

- removing false matches (outliers);

- calculating parameters of geometric transformations;

- transforming VTM image.

In this work we used well-known methods for feature extraction, such as SIFT (Scale Invariant Feature Transform) [1], SURF (Speeded Up Robust Features) [5] and ORB (Oriented Binary Robust Independent Elementary Features) [4, 6]. Matching of descriptors was implemented by Brute-Force and FLANN based matchers [6]. Usually, a main drawback of feature points is the large number of false positive detections. This drawback can be overcome by the use of hypothesis rejection methods, such as RANSAC [2]. Calculation of parameters of the geometric transformation is an important task of the algorithm, as it will depend on the quality of registration performed. Solution of this problem can be conducted in two ways: finding affine or perspective transform parameters $[3,6]$. Generally these parameters are the combination of scale, bias and rotation.

\subsection{Correction using searching registration}

The causes of blunders can be the movement of the video sensor, the differences between the current and the base frame, the improper registration of searching algorithm. In order to improve the results it is necessary to protect them against the emergence of erroneous registration. To predict this situation, the opportunity of amendment by searching registration is used. The result of its work is the replacement of the base frame and clarifying the image of VTM. Correction must be agreed with the automatic solution. Variables for setting criteria can be: the residual norm, the estimation shift between centers of two TV images, the share dropped anchor points, number of matches. According to the experimental results, the best criteria is shift between two centers of the images. The typical situation is presented on fig.2. This screenshot corresponds with a vertical line on fig. 3 .

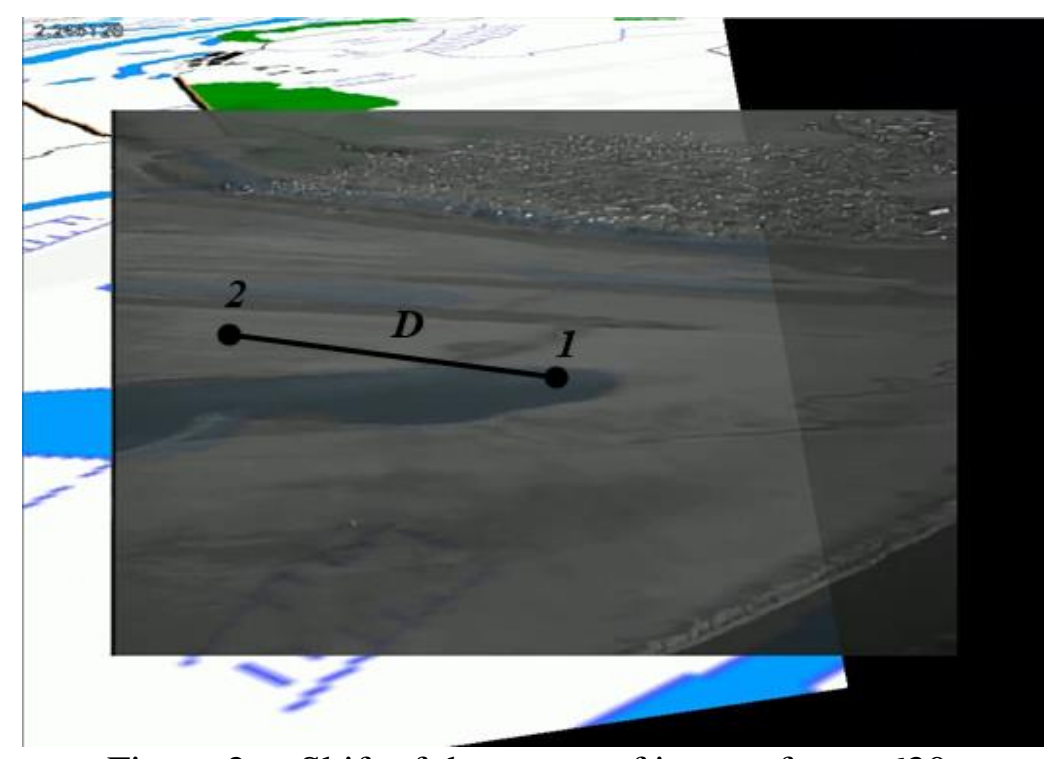

Figure 2. - Shift of the center of image, frame 630.

Point 1 with coordinates $\left(x_{1}, y_{1}\right)$ on fig. 2 corresponds to the centre of base image, point 2 with coordinates $\left(x_{2}, y_{2}\right)$ - centre of current image. The distance between them can be found easily:

$$
D=\sqrt{\left(x_{2}-x_{1}\right)^{2}+\left(y_{2}-y_{1}\right)^{2}}
$$




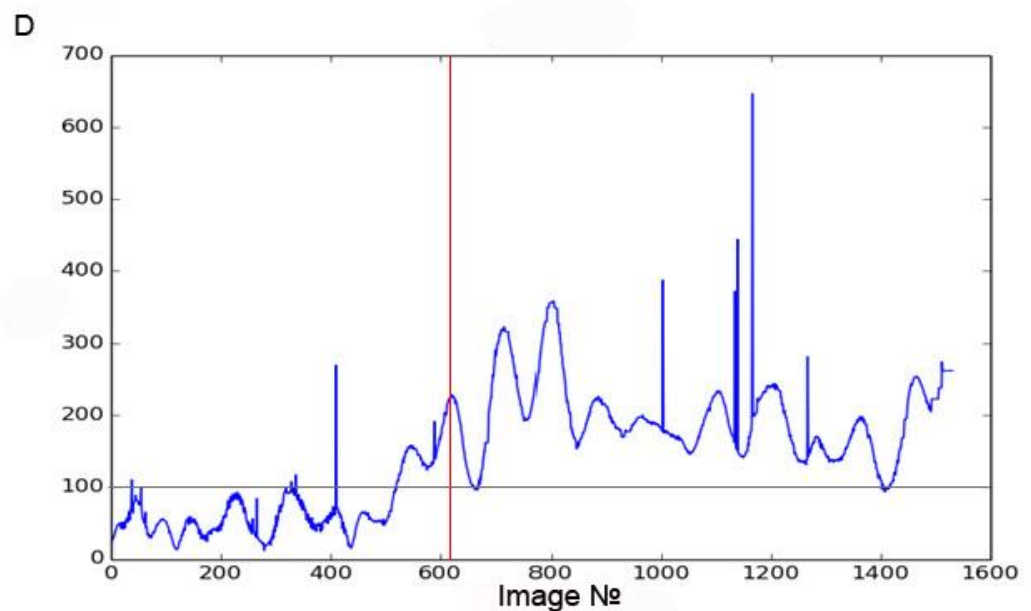

Figure 3. - Shift plot.

If distance $D$ is more than 100 for five frames, according to this rule searching registration starts. Single false bursts can be compensated by using geometric transformation parametres from the previous frame.

\section{Experimental Results}

Mentioned algorithms were investigated in experimental researches. Performance was evaluated on a software module using test videos. Debugging was carried out on a PC with the following specifications: processor - Intel (R) Core (TM) 2 Duo E7400, 2.80GHz (2 CPUs); amount of RAM - 4 GB.

SIFT, SURF, ORB OpenCV implementations were used [7]. We have evaluated the proposed algorithm written in $\mathrm{C}++$ and compared the results to the searching registration, using only CEA. The average time spent for a single frame is shown in table 1.

Table 1. - Time indexes

\begin{tabular}{|c|c|c|c|c|}
\hline \multirow{2}{*}{ Video } & \multicolumn{4}{|c|}{ Time, sec } \\
\cline { 2 - 5 } & SURF & SIFT & ORB & CEA \\
\hline 1 & 0.229 & 0.571 & 0.052 & 5.539 \\
\hline 2 & 0.106 & 0.551 & 0.045 & 4.998 \\
\hline 3 & 0.213 & 0.578 & 0.057 & 5.544 \\
\hline
\end{tabular}

As you can see the proposed algorithm can significantly speed-up the registration process. It also improves quality of the alignment. As we do not have the original reference data for evaluation, this confirmation may be obtained from the plots of rotation of the VTM image.

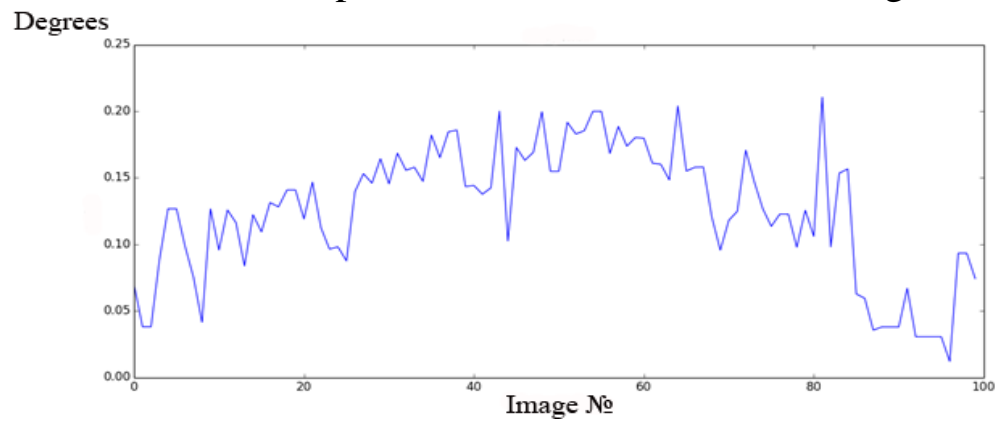

Figure 4. - Rotation of the VTM image using proposed algorithm. 


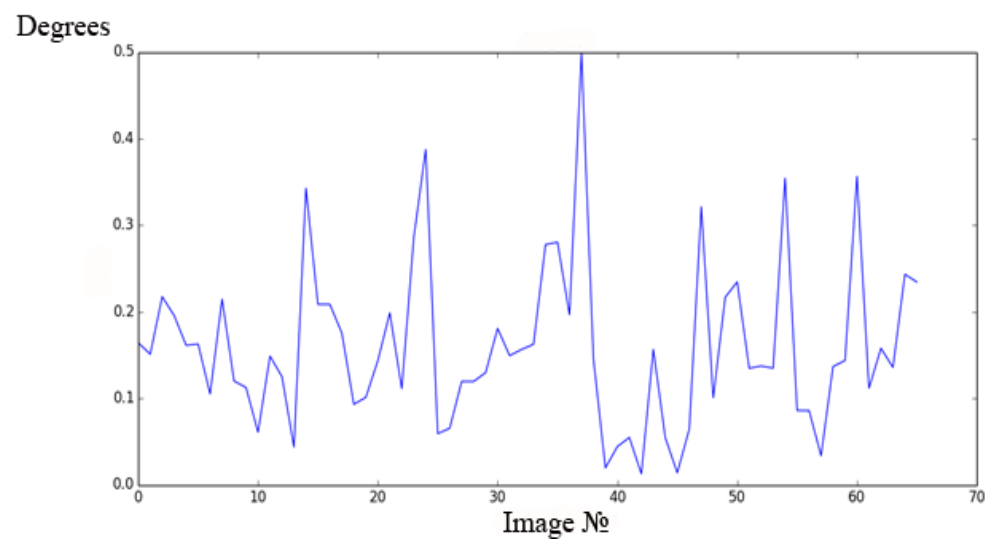

Figure 5. - Rotation of the VTM image using CEA.

Fig. 4 illustates the rotation of the VTM image using the proposed algorithm. As it shows, rotation changes insignificantly and smoothly between the frames. In front of the situation on fig. 5. The source video sequence was recorded during smooth sensor movement. Therefore, fig. 4 better conforms with the source image data. With this in mind, we can present it as an advantage of the proposed approach.

Finally, fig. 6 shows an example of situation, when proposed algorithm stands better with the problem of registration than CEA.

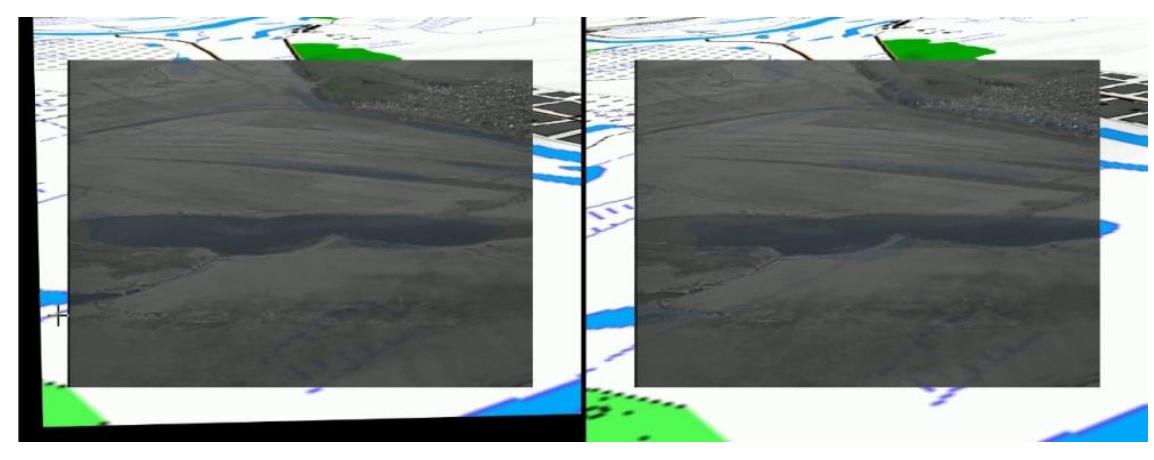

a)

b)

Figure 6. - Image registration: a) - proposed algorithm; b) - original algoritm based on CEA.

\section{Conclusions}

After research analysis we can conclude that the developed approach is generally superior to the approach based on CEA. The main advantage is fast image processing, so for the whole image registration average time is reduced in 40-50 times. In this way, combined using searching and tracking techniques is an effective way to solve the problem of image registration.

\section{Acknowledgements}

This work was supported by grant RFMEFI57414X0056 from the Ministry of Education and Science of the Russian Federation (contract №14.574.21.0056).

\section{References}

[1] Manuele Bicego, Andrea Lagorio, Enrico Grosso, and Massimo Tistarelli. On the use of SIFT features for face authentication. In IEEE International Conference on Computer Vision and Pattern RecognitionWorkshop (CVPRW), New York, USA, 2006. 
[2] M.A. Fischler and R.C. Bolles. Random sample consensus: A paradigm for model fitting with applications to image analysis and automated cartography. Communications of the ACM, June 1981.

[3] David G. Lowe. Distinctive image features from scale-invariant keypoints. International Journal of Computer Vision (IJCV), 2004.

[4] Rublee E., Rabaud V., Konolige K., Bradski G. ORB: an efficient alternative to SIFT or SURF. Proceedings of 13th International Conference on Computer Vision, Barcelona. 2011.

[5] Bay H., Ess A., Tuytelaars T., Van Gool L. SURF: Speeded Up Robust Features. Proceedings of 9th European Conference on Computer Vision (ECCV), Graz (Austria), 2006.

[6] Official OpenCV documentation (2D Features Framework): http://docs.opencv.org/modules/features2d/doc/features2d.html

[7] Official web-site of OpenCV library(Open Source Computer Vision): http://opencv.willowgarage.com/wiki

[7] Alpatov B., Korepanov S., Strotov V. A composite algorithm for variable size object tracking for high performance FPGA-based on-board vision systems //SPIE Remote Sensing. International Society for Optics and Photonics, 2014.

[8] Alpatov B.A., Strotov V.V. An estimation algorithm of the multispectral image geometric transformation parameters based on multiple reference area tracking // SPIE Defense, Security, and Sensing. - International Society for Optics and Photonics, 2013.

[9] Alpatov, B.A., Babayan, P.V., Balashov, O.E. and Stepashkin, A.I. The methods of the automatically object detection and tracking. Image processing and control, Radiotechnika, Moscow (2008). 